\title{
Whither pulmonary rehabilitation? Will alternative modes help or hurt?
}

\author{
Richard Casaburi
}

Affiliation: Rehabilitation Clinical Trials Center, Los Angeles Biomedical Research Institute at Harbor-UCLA Medical Center, Torrance, CA, USA.

Correspondence: Richard Casaburi, Rehabilitation Clinical Trials Center, Los Angeles Biomedical Research Institute at Harbor-UCLA Medical Center, 1124 W Carson Street, Torrance, CA 90502, USA.

E-mail: casaburiducla.edu

@ERSpublications

Pulmonary rehabilitation is available to only a sliver of COPD patients. Studies have explored less resource intensive approaches. Unless they prove to be at least as effective, traditional pulmonary rehabilitation availability may be further reduced. http://ow.ly/fyh930lYFo2

Cite this article as: Casaburi R. Whither pulmonary rehabilitation? Will alternative modes help or hurt? Eur Respir J 2018; 52: 1801678 [https://doi.org/10.1183/13993003.01678-2018].

Chronic obstructive pulmonary disease (COPD) causes great misery to those afflicted. While it would be nice to think that a cure is near at hand, this remains only a distant dream. Symptom relief is the best we can do for the time being. We look for therapies to provide important patient-centred benefits such as improved exercise tolerance, reduced dyspnoea and better health-related quality of life. When we consider available therapies, we might consider comparing the magnitude of benefits of bronchodilators with those of pulmonary rehabilitation. A recent informal comparison of these benefits gleaned from meta-analyses in the literature reveals that, for exercise tolerance, dyspnoea and quality of life, the yield of pulmonary rehabilitation is several-fold greater than for bronchodilators [1]. In this analysis, pulmonary rehabilitation benefits well exceed thresholds of clinical importance in all three of these domains. Moreover, over the past 25 years, pulmonary rehabilitation has gained a bulletproof physiological basis $[2,3]$; the strategies employed, especially in the area of exercise training, are highly evidence-based.

Despite this, pulmonary rehabilitation is only available to a small sliver of COPD patients who could benefit $[4,5]$, as compared to the almost universal availability of bronchodilator therapy. The reasons for this discrepancy are hard to understand, since both therapies receive strong international recommendation as standard of care [6] and some analyses judge pulmonary rehabilitation to have higher cost-effectiveness than bronchodilator therapy [7].

Those of us in the pulmonary rehabilitation research community have been conducting a frantic search for strategies to expand the availability of pulmonary rehabilitation benefits [8]. One highly reasonable approach has been to seek modes of rehabilitative therapy that differ from the traditional model: in-centre programmes run by a multi-disciplinary team of rehabilitation professionals.

In this light, the novel and innovative intervention designed and reported by ARBILLAGa-ETXARri et al. [9] in this issue of the European Respiratory Journal is very interesting. Though not specifically promoted as such, their "Urban Training" programme is a rehabilitative intervention. It has the virtue of requiring neither the extensive facilities nor the focused attention of a rehabilitative team, features that are inherent in the traditional pulmonary rehabilitation model. The primary outcome was physical activity level, an important outcome in the sedentary COPD population. Similar to the findings of studies of pulmonary rehabilitation [10], the activity level changes were present in only a subset of participants, in this case 
those that complied with the intervention. It deserves to be mentioned that those assigned to the Urban Training programme, but who for whatever reason failed to comply, actually experienced an appreciable decrease in activity level. An important finding among the secondary outcomes is that participants in the Urban Training programme improved neither a measure of exercise tolerance nor measures of quality of life.

This study has a number of lessons. One is that novel approaches, tailored to specific environments by a talented group of investigators, can demonstrate important benefits, at least in a subset of COPD participants. Another, though, relates to a larger topic: that of the role of alternative modes of rehabilitative therapy in COPD. It can be posited that promoting alternative modes will further disincentivise provision of traditional pulmonary rehabilitation programmes. But a corollary of this proposition is that we must determine whether these alternative modes provide equivalent benefits. Certainly, if a rehabilitative strategy, that is simpler and cheaper to deliver, can be shown to have equivalent (or superior) benefits, then we can joyfully allow traditional pulmonary rehabilitation to wither away. But if these alternatives have inferior outcomes, the damage to traditional programmes could be considerable.

It becomes important to have metrics against which to gauge the success of these alternative interventions. One strategy is to employ a control group that engages in a traditional rehabilitation programme and compare the outcomes with the group receiving the novel intervention. But this metric, too, may yield difficult to interpret results. Consider the study of Holland et al. [11], which concluded that "home-based pulmonary rehabilitation ... produced short-term clinical outcomes that were equivalent to centre-based pulmonary rehabilitation". True, the exercise tolerance improvement, assessed by 6-min walk distance (the primary outcome), was non-inferior (and tended to be superior) in the home-based group as compared to the in-centre group. However, the improvement in the in-centre group was only $11 \mathrm{~m}$, well less than the 46- $\mathrm{m}$ average improvement seen in a meta-analysis of traditional pulmonary rehabilitation programmes [12]. A second example is the recently-published study of Polkey et al. [13], who compared the benefits of Tai Chi with traditional rehabilitation in a group of rural Chinese farmers with COPD who were naïve to bronchodilator treatment. It was concluded that "Tai Chi is an appropriate substitute for pulmonary rehabilitation". Here, too, 6-min walk distance in both the Tai Chi and traditional rehabilitation control group (roughly 21-m increases) were somewhat lower than the meta analysis would lead us to expect [12]. True, the improvement in quality of life assessed by the St. George's Respiratory Questionnaire (SGRQ, the primary outcome measure) was substantial in both study arms (about 16 points). But a 2-week run in period, in which all subjects were initiated on long-acting beta-agonist bronchodilator therapy, yielded a similarly large decrease in SGRQ ( $\sim 16$ points); much larger than has ever been seen with a bronchodilator intervention [14]. This unexpected result raises the possibility that the quality of life results are not generalisable to the broader COPD population.

In the future, I would hope that results of these novel interventions would be gauged not only against an appropriate control group, but also against established metrics based on the extensive traditional pulmonary rehabilitation literature. These comments are not intended to denigrate the efforts of fine researchers who to attempt advance the field of pulmonary rehabilitation in this way, but only to point out the risk of disincentivising a (likely) superior therapy.

In the past few years, studies have appeared testing the effectiveness of, for example, tele-rehabilitation and home rehabilitation. Yet I wonder whether their equivalence to traditional in-centre programmes is even plausible. The deep-seated sedentary behaviour of most COPD patients is hard to reverse without the sustained effort of professionals trained in motivational techniques and the support of similarly afflicted patients in group sessions; these are key features of traditional rehabilitation.

Are there other approaches to expanding the availability of pulmonary rehabilitation that should be encouraged? First, we can lobby for increased reimbursement. In the USA, pulmonary rehabilitation is reimbursed at half the rate of cardiac rehabilitation. Financial unsustainability is causing a substantial number of US programmes to close. Second, we can harness the "silent millions" of COPD patients. The COPD Foundation's "COPD 360 Social" [15], an online patient forum with a membership of more than 35000 from over 130 countries, is a model of what can be done to give COPD patients a voice. Third, delivery of pulmonary rehabilitation requires a group of dedicated well-trained professionals. Specific training programmes in pulmonary rehabilitation are lacking in many countries; their establishment should be promoted. Finally, many pulmonary rehabilitation advocates (including me) believe that pulmonary rehabilitation prolongs life. Life-prolonging therapies have a special place in our hierarchy of therapies. Whether pulmonary rehabilitation prolongs life has never been adequately tested [16, 17]; such a clinical trial should have a high priority. 


\section{References}

1 Casaburi R. Pulmonary rehabilitation: where we've succeeded and where we've failed. COPD 2018; in press [https://doi.org/10.1080/15412555.2018.1503245]

2 Maltais F, Decramer M, Casaburi R, et al. An official American Thoracic Society/European Respiratory Society statement: update on limb muscle dysfunction in chronic obstructive pulmonary disease. Am J Respir Crit Care Med 2014; 189: e15-e62.

3 Casaburi R, Porszasz J, Burns MR, et al. Physiologic benefits of exercise training in rehabilitation of patients with severe chronic obstructive pulmonary disease. Am J Respir Crit Care Med 1997; 155: 1541-1551.

4 Desveaux L, Janaudis-Ferreira T, Goldstein R, et al. An international comparison of pulmonary rehabilitation: a systematic review. COPD 2015; 12: 144-153.

5 Nishi SP, Zhang W, Kuo YF, et al. Pulmonary rehabilitation utilization in older adults with chronic obstructive pulmonary disease, 2003 to 2012. J Cardiopulm Rehabil Prev 2016; 36: 375-382.

6 Global Strategy for the Diagnosis, Management and Prevention of COPD. Global Initiative for Chronic Obstructive Lung Disease (GOLD) 2018. https://goldcopd.org/gold-reports/ Date last accessed: September 2, 2018.

7 Zoumot Z, Jordan S, Hopkinson NS. Emphysema: time to say farewell to therapeutic nihilism. Thorax 2014; 69: 973-975.

8 Rochester CL, Vogiatzis I, Holland AE, et al. An Official American Thoracic Society/European Respiratory Society Policy Statement: enhancing implementation, use, and delivery of pulmonary rehabilitation. Am J Respir Crit Care Med 2015; 192: 1373-1386.

9 Arbillaga-Etxarri A, Gimeno-Santos E, Barberan-Garcia A, et al. Long-term efficacy and effectiveness of a behavioural and community-based exercise intervention (Urban Training) to increase physical activity in patients with COPD: a randomised controlled trial. Eur Respir J 2018; 52: 1800063.

10 Cindy $\mathrm{Ng}$ LW, Mackney J, Jenkins S, et al. Does exercise training change physical activity in people with COPD? A systematic review and meta-analysis. Chron Respir Dis 2012; 9: 17-26.

11 Holland AE, Mahal A, Hill CJ, et al. Home-based rehabilitation for COPD using minimal resources: a randomised, controlled equivalence trial. Thorax 2017; 72: 57-65.

12 McCarthy B, Casey D, Devane D, et al. Pulmonary rehabilitation for chronic obstructive pulmonary disease. Cochrane Database Syst Rev 2015;2: CD003793.

13 Polkey MI, Qiu ZH, Zhou L, et al. Tai Chi and pulmonary rehabilitation compared for treatment-naive patients with COPD: a randomized controlled trial. Chest 2018; 153: 1116-1124.

14 Kew KM, Dias S, Cates CJ. Long-acting inhaled therapy (beta-agonists, anticholinergics and steroids) for COPD: a network meta-analysis. Cochrane Database Syst Rev. 2014;3: CD010844.

15 Stellefson M, Paige SR, Alber JM, et al. COPD360social Online Community: a social media review. Health Promot Pract 2018; 19: 489-491.

16 Puhan MA, Gimeno-Santos E, Cates CJ, et al. Pulmonary rehabilitation following exacerbations of chronic obstructive pulmonary disease. Cochrane Database Syst Rev 2016; 12: CD005305.

17 Troosters T, Casaburi R, Gosselink R, et al. Pulmonary rehabilitation in chronic obstructive pulmonary disease. Am J Respir Crit Care Med 2005; 172: 19-38. 